\title{
Comparison between Femtosecond Assisted LASIK and Small Incision Lenticule Extraction (SMILE) for Correction of Myopic Astigmatism
}

\author{
Amr Sheta ${ }^{1}$ MSc; Mahmoud Ismail ${ }^{2}$ MD; Abd El-Magid Tag El-Din ${ }^{2}$ MD and Ahmed El-Shahed ${ }^{3}$ MD.
}

\section{* Corresponding Author: \\ Amr Sheta \\ Shetaamr@yahoo.com}

Received for publication April 26, 2020; Accepted August 25, 2020; Published online August 25, 2020.

Copyright 2020 The Authors published by Al-Azhar University, Faculty of Medicine, Cairo, Egypt. All rights reserved. This an openaccess article distributed under the legal terms, where it is permissible to download and share the work provided it is properly cited. The work cannot be changed in any way or used commercially.

doi: $10.21608 /$ aimj.2020.28754.1206

${ }^{1}$ Ophthalmology Department, Military medical collage.

${ }^{2}$ Ophthalmology Department, Faculty of Medicine, Al-Azhar University.

${ }^{3}$ Ophthalmology Department Faculty of Medicine, Helwan University.

\begin{abstract}
Background: In the field of refractive surgery, femtosecond laser has gained favorable results for use in the creation of corneal flaps, with much more decrease of flap complications. Being a flapless procedure, FS-SMILE was introduced to avoid the flap complications.

Aim of the work: To evaluate Fs-LASIK and SMILE in phrases of efficacy, safety, predictability. Also, an assessment the impact of each technique in high-order corneal aberrations, corneal asphericity and eye dryness.

Patients and Methods: prospective, non-randomized, comparative clinical trial was done from June 2018 to November 2019 on myopic patient. This test consists of 60-eyes that were operated at El Mashreq Eye Hospital. Fs- LASIK group covered 30 -eyes and SMILE group covered 30-eyes.

Results: No-statistically significant difference between both maneuvers in efficacy, safety and predictability. $86.61 \%$ in the SMILE group, and 93.32\% eyes in the FS-LASIK group, respectively, had 20/20 or better UDVA. Dry eye was manifested in less degree after SMILE procedure compared with Fs-LASIK. Both Fs-LASIK and Fs-SMILE increased total corneal coma aberration and spherical aberrations, but there has been a non-statistically large extension between each strategy. Smile possibly brought on a higher coma aberration due to decentration and fsLASIK resulted in more spherical aberration.

Conclusion: The results in both procedures are matching and comparable but a further contralateral eye studies is needed to have a homogenous preoperative matching and avoid bias.
\end{abstract}

Keywords: Femtosecond Assisted LASIK; Lenticule Extraction (SMILE); Myopia.

Disclosure: The authors have no financial interest to declare in relation to the content of this article. The Article Processing Charge was paid for by the authors.

Authorship: All authors have a substantial contribution to the article.

\section{INTRODUCTION}

LASIK is the best-known refractive procedure in the world to treat myopia and astigma. However, the induction of HOAs is an issue following LASIK. ${ }^{1}$

The main challenges include predictability, long-term correction fixability, visual quality, safety, and the biomechanical balance of the cornea with further complications. ${ }^{2}$

Femtosecond laser (FL) induces precise cavitation of the ocular tissues and is used for the development of corneal flaps. FL was recently used to generate an clear refractive lenticule with lifted corneal flap (FLEx) or without lifted flap as in (SMILE). ${ }^{3}$

The HOAs were exaggerated after the procedures, while the FL was improved. The development of corneal flaps and localized inflammation will be key to promoting this changes. ${ }^{4}$
Fs-LASIK demonstrated its power, stability and predictability, as one of the most effective corneal refractive methods. Nonetheless, there are some problems restricting Fs-LASIK software, which includes the possibility of complications associated with flaps and dry eyes. ${ }^{5}$

Some side effects couldn't be avoided, like astigmatism, corneal resistance factor (CRF), changes of corneal hysteresis $(\mathrm{CH})$, photaesthesia or higher order aberration (tHOAs), due to the damaged corneal stromal floor. ${ }^{6}$

For myopic treatment and reduced risk of postoperative corneal dryness, supposed post-operative corneal disease SMILE was applied first in 2008 for overly successful, reliable, balance, and protection. ${ }^{7}$

SMILE approach minimizes postoperative inflammatory and wound healing response as compared to Fs-LASIK. ${ }^{8}$ 
SMILE damages less corneal nerves and preserves corneal biomechanics in evaluation with Fs-LASIK. Many different conclusions were drawn concerning the visual quality postoperative between the two maneuvers, especially biomechanical results. ${ }^{10}$

\section{PATIENTS AND METHODS}

A prospective, non-randomized, comparative clinical trial was done on 60-eyes which have been recruited from El Mashreq Eye Hospital with myopia or myopic astigmatism provided for refractive errors correction. Patients have been divided into two groups: Group (A); covered (30) eyes underwent SMILE approach. Group (B); covered (30) eyes underwent Fs-LASIK. Criteria for inclusion; age 18 years and older, manifest refraction spherical equivalent (MRSE), no more than $8 \mathrm{D}$, no more than 3D refractive cylinder, no topographic evidence of forme fruste keratoconus (FFKC) and no more than $0.5 \mathrm{D}$ for refractive exchange in the last 12 months. Criteria for exclusion: previous eye damage or procedure, scars and opacity of the cornea, recurrent eye infection like uveitis and glaucoma, processes affecting recovery and healing such as diabeted mellitus, vascular collagen disorders and dry eye conditions.

Pre-operative evaluation: Uncorrected and corrected distance visual acuity, refraction, IOP, slit lamp and fundus examination, testing of dry eye using Schirmer test and tear break up time check (TBUT), corneal topographic analysis, and wave-front analysis using combined Schimpflug and placido disc.

Immediate preoperative counseling: The cases can be recommended about the manner, sounds and steps. Anxious cases may be given an oral sedative.

Preoperative medications: Topical anesthesia (Benoxinate 4\% eye drops. three times, 5 minutes apart). Povidone-iodine (10\% to prep the skin \& $5 \%$ drops to the ocular floor), left for 30 seconds then irrigated.

FS-LASIK: Using topical anesthesia and draping lid speculum. The VisuMax 500 (Carl Zeiss, Inc.) was used to shape a superiorly hinged $110-\mu$ m thick flap. With inverted side cut (70). WaveLight MEL90 (Carl Zeiss, Inc.) was subsequently applied to excimer laser ablation, usage the LASIK aspheric profile Q-adjusted. Following ablation, the flap is repositioned carefully and medication after surgical procedure started.

SMILE: The eye of the selected individuals is oriented and docked under topical anesthesia, large sterile draping and insertion of the speculum. During the following series, the laser has been modified: first, the back floor of the refractive lenticula and then the lenticula boundary. The front floor of the refractive lenticule was then rendered and the rim cut was followed. Thickness $[100 \mu \mathrm{m}]$, diameter $[7.70$ $\mathrm{mm}$ ], width of the incision [3 $\mathrm{mm}$ ], incision angle $\left[120^{\circ}\right]$, the following FS parameters are used for the cap. The FS parameters for lenticule have been used: Side-cut angle $\left[120^{\circ}\right.$ ],thickness [Min:10 $\mu \mathrm{m}$, Max: 155 microns], [0.10 mm] sector of transition, [6.20 $\mathrm{mm}$ ] optical sector. After the suction was removed, a Siebel spatula was inserted below the cap close to the hinge before the cap was dissected. The edge of lenticule was separated from the stromal bed with a sinsky hook and the posterior border of the lenticule come to be gently separated with spatula. The lenticule was holded and removed with serrated forceps through the small incision

Postoperative treatment: Topical eye drops of tobradex and dexamethasone were used every 4 hours during 1 week after surgery. In addition, topical lubricants were recommended for 3 months every four hours.

Postoperative evaluation: Immediately postoperative, 1st day post-operative (slit-lamp Exam), 1st week post-operative, 1st month and 3rd month post-operative (identical as pre-op).

Expected primary outcome measures: UCVA \& BCVA, manifest refraction, refractive predictability, effectiveness (efficiency $=$ UCVA / BCVA preoperational percentage), safety: $($ Safety $=$ BCVA post-operative / BCVA preoperative percentage).

Expected secondary outcome measures: To compare and test the effect of Fs-LASIK and SMILE on corneal dryness, optical quality and HOAs.

Statistical assessment: The MedCalc version 12.7.0 was used for all statistical analyzes.

\section{RESULTS}

Among 30 cases, myopia and myopic astigmatism has been subjected to refractive surgical therapy. It was made SMILE with 30 eyes and Fs-LASIK with 30 eyes (Figure 1). Patients were among 20 and 40 years of age. There were no significant difference regarding age $(\mathrm{P}=0.115)$, UDVA $(\mathrm{P}=0.8736)$, CDVA $(\mathrm{P}=1.00)$ or SE $(\mathrm{P}=0.6914)$ between the two groups preoperatively.

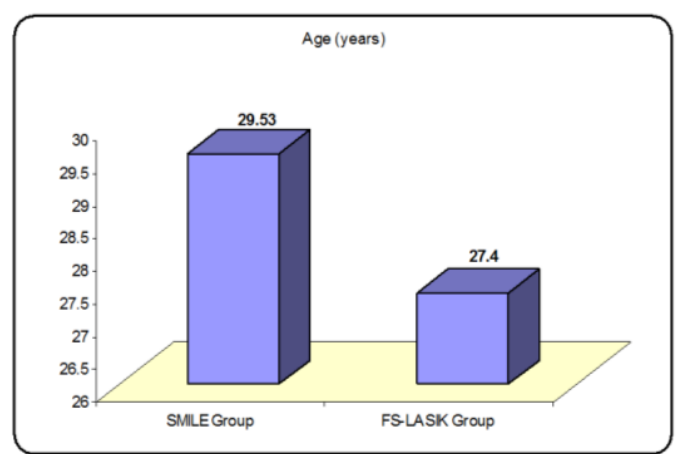

Fig. 1: Age Distribution among SMILE \& Fs-LASIK Groups. 


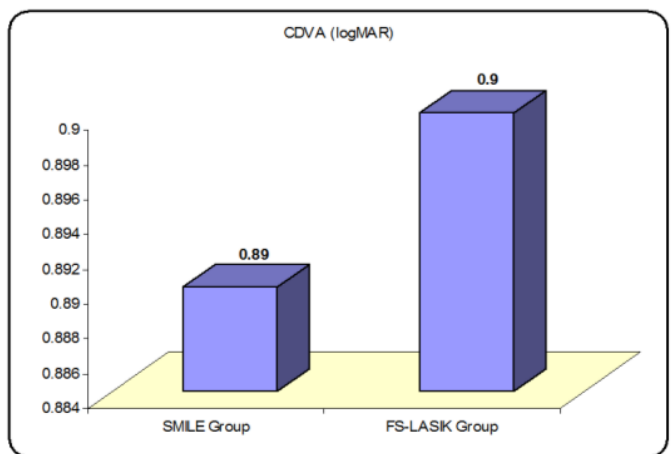

Fig. 2: UDVA Distribution among in SMILE \& FsLASIK Groups.

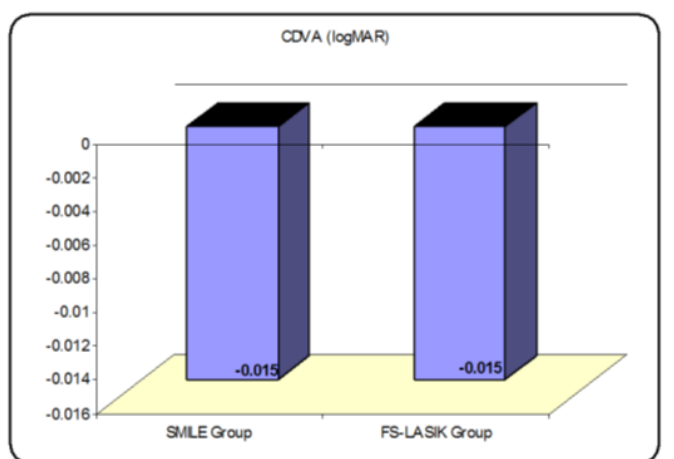

Fig. 3: CDVA Distribution among SMILE \& FsLASIK Groups.

Refraction: In the SMILE group, the mean MRSE 3 months later was: $-0.2463 \pm 0.377 \mathrm{D}$, while the average MRSE of the Fs-LASIK Group was 0.3278 $\mathrm{D}(-1.25-0.5 \mathrm{D})$. There were no major statistical differences between two groups $(\mathrm{P}=0.4975)$.

Predictability: $93 \%$ of each group had been within \pm $0.25 \mathrm{D}$ of achieved \& attempted correction after 3 months. Also, $95.67 \%$ and $100 \%$ of eyes in the SMILE and FS-LASIK groups, had been within \pm 1 $\mathrm{D}$, respectively.

Effectiveness: $86.61 \%$ of SMILE patients, and $93.32 \%$ of Fs-LASIK had 20/20 or higher UDVA after 3 months of surgery. All handled eyes in every SMILE \& FS-LASIK groups had 20/20 or better UDVA. A non-statistically difference of UDVA was located among SMILE group $(-0.025 \pm 0.09446)$ and FS-LASIK groups $(-0.04 \pm 0.09861)(\mathrm{P}=0.5774)$. The efficacy index turned into $1.0245 \pm 0.1688$ within the SMILE and $1.068 \pm 0.1976$ within the FS-LASIK group and there has been no statistically big distinction between both of them $(\mathrm{P}=0.3888)$

Safety Outcomes: There were no improvement in 11 (36.67\%) eyes in the SMILE group 3 months later regarding the CDVA, $14(46.67 \%)$ eyes acquired 1 line, $1(3.33 \%)$ eyes received more than one line, and $3(10 \%)$ lost one line, while $13(43.66 \%)$ eyes had no alternate in the FS-LASIK group, 12 (30\%) had won 1 line, $4(13.33 \%)$ had gained more than a line, respectively. There is no statistically significant postoperative variation of CDVA in all groups $(\mathrm{P}=$ $0.5287)$. In the SMILE group, the safety index rose to $1.0953 \pm 0.1368$ and within the Fs-LASIK group $1,1224 \pm 0,153$. The safety index did not have a statistically significant difference $(\mathrm{P}=0.5199)$ between the two groups.

Dry eye parameters: For both of the schirmer-1 test $(\mathrm{P}=0.171)$ and TBUT $(\mathrm{P}=0.0081)$ in the SMILE Group at 3 months postoperatively; statistically non- significant difference was found. In FS - LASIK, each schirmer-1 $(\mathrm{P}<0.0001)$ and TBUT $(\mathrm{P}<0.0001)$ test was statistically significantly decreased. At 3 months after surgery, the statistically significant difference was between Schirmer 1 and TBUT. (Figures 4, 5).

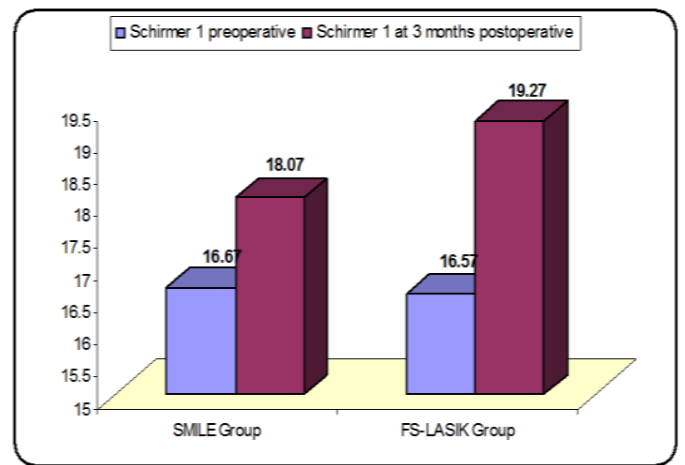

Fig. 4: Schirmer-1 for SMILE and Fs-LASIK.

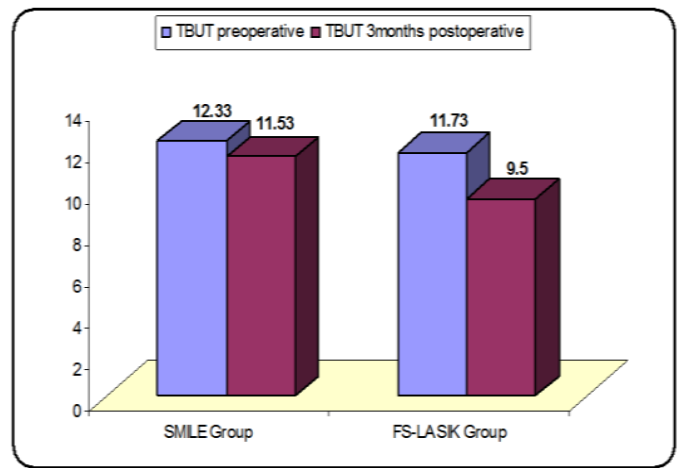

Fig. 5: TBUT for SMILE and Fs-LASIK.

Corneal asphericity changes: It has become measured at $6 \mathrm{~mm}$ diameter for all patients. In SMILE group, the mean $Q$ value preoperatively have become -0.104 (SD: \pm 0.1354 , variety $(0.21$ to -0.32$)$ then 0.022 (SD: \pm 0.3085 , variety $(0.63$ to -0.65$)$ postoperatively. We observed a statistically extraordinary growth in $\mathrm{Q}$ value postoperatively $(\mathrm{P}=$ 0.0176). In Fs-LASIK, the mean $Q$ value preoperatively turn out to be -0.142 (SD: \pm 0.1275 , range $0.06-0.49$ ) then 0.063 (SD: \pm 0.1414 , variety (0.4 to -0.12) postoperatively. A statistically notable growth in $\mathrm{Q}$ value postoperatively $(\mathrm{P}<0.0001)$ was noticed. The difference in $\Delta \mathrm{Q}$ was not statistically significant between the two maneuvers. Further Oblate anterior surface was done by Fs-LASIK ( $\mathrm{P}=$ 0.2124). (Figure 7) 


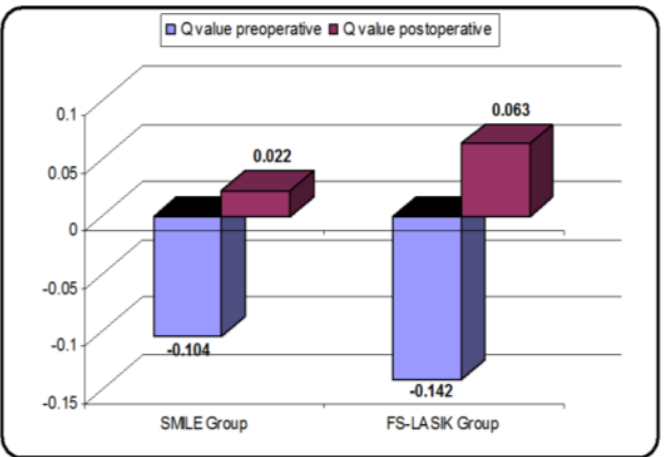

Fig. 6: Difference of preoperative, postoperative and $\mathrm{Q}$ values between the two groups.

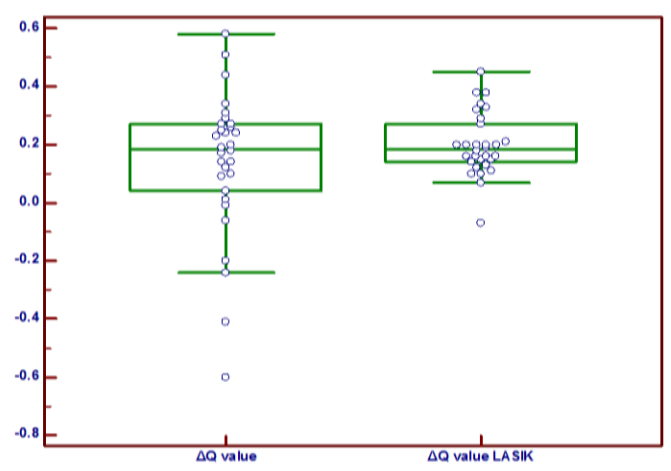

Fig. 7: · Adjust Q value following SMILE and FSLASIK.

Corneal high order aberrations: In SMILE the RMS of total corneal aberration $(\mathrm{P}=0.0003)$, coma aberration $(\mathrm{P}=0.0003$, t-test $)$, and spherical aberrations $(\mathrm{P}=$ null.0434 $)$ were statistically significantly increased. In the case of Fs-LASIK, total corneal aberration $(\mathrm{P}<0.0001)$, coma aberration $(\mathrm{P}=0.0010)$ ), and spherical aberrations $(\mathrm{P}$ $<0.0001)$ has been statistically significantly increased in RMS. In comparing both groups, there has been no statistically large distinction inside the exchange in RMS of total corneal high order aberration $(\mathrm{P}=0.2000)$, coma aberration $(\mathrm{P}=$ $0.0589)$ and spherical aberration $(\mathrm{P}=0.0543)$. However, SMILE confirmed more change in total aberration and coma aberration. While, Fs-LASIK showed more alternate in spherical aberration.

\section{DISCUSSION}

SMILE \& FS-LASIK were extremely effective, safe and predictable.. The effectiveness and safety indices for SMILE \& Fs-LASIK were identical. ${ }^{\mathbf{1 2}}$

Regarding UDVA of 20/20 or higher, we placed results just like the ones of previous research with SMILE $^{13}$ and Fs-LASIK. ${ }^{12}$ Concerning CDVA and quality, dry eye is probably the main cause for decreasing of CDVA. ${ }^{14}$ As far as predictability is concerned, every SMILE and FS-LASIK had a three months excessive emeteropia tendency. However, Vestergaard et al. ${ }^{4}$ confirmed, that SMILE was in the direction of emmetropia more than FS-LASIK. Upon activity, the refraction among both groups that fulfill Gertnere et al. ${ }^{15}$ was non-significant different. Dry eye condition is an acute post-refractive issue because the eye comfort determines its satisfaction. ${ }^{16}$ The dry eye disorder is a multifactorial eye surface disorder involving changes in the tear film and an improvement in tear osmolarity with or without the corneal injury. ${ }^{17}$

Golas et al. ${ }^{18}$ suggested that the tear secretion characteristic have become suppressed within the course of the first 3 months and returned back to normal levels with the beneficial resource of 6 months after surgical treatment, whilst HorwathWinter, et al. ${ }^{19}$ confirmed that Schirmer test results had no variations. Toda et al. ${ }^{20}$ concept that dry eye symptoms become parallel to that of corneal sensitivity. LASIK-precipitated dry eye is based mainly, on the breakdown of sub-basal nerves In addition, the SMILE protects the innervation of the cornea by producing just a small penetration tunnel for 40 to 60 degree in contrast to the wide penetrating tunnel for LASIK, around 300 degree. Reports have accepted reduced corneal sensitivity following LASIK, which is likely to last months or maybe years while a Fs laser is used in the manufacturing of flaps. ${ }^{21}$ Further information about mechanisms in post-refractive dry eye disease pathogenesis is a big issue. $^{22}$

The induction of giant quantities of HOAs has been defined as a crucial side impact. ${ }^{23}$ It was related to a number of factors including biomechanics of the cornea, ablation algorithms, flap formation and ablation decentration. ${ }^{24}$ The use of the Fs-laser still delivered about ordinary HOAs, coma and spherical aberrations. No statistically huge difference among groups in RMS of established corneal higher order aberrations, with more induction of coma aberration in SMILE group and spherical aberrations in FsLASIK age. For qualitative purposes, Ye, et al. ${ }^{25}$ Vestergaard et al. 4 and Gertnere et al ${ }^{15}$ agreed with SMILE that the marginal growth in spherical aberration relative to the Fs-LASIK was simultaneously seen.

Spherical aberration in myopic patients with a greater pupil diameter caused more problem than coma-likes aberration. ${ }^{26}$ Our literature was used in the aspheric ablation model in SMILE and Fs - LASIK groups, so that corneal curvature did not lead to any major spherical aberration changes. The formation of a flap, however, is another important explanation for HOAs. ${ }^{27}$ By maintaining the biomechanical balance, SMILE minimizes the alternative form of a cornea. ${ }^{28}$ In addition, spherical post-operative aberration correlating with optical and ablation zones. ${ }^{29}$ Coma aberration is greater with SMILE, as it does no longer encompass the iris registration technology. In addition, the SMILE concentration is much less accurate than the excimer laser attention tracker. ${ }^{30}$ Furthermore, coma aberration represents irregularity, propensity and concentration. ${ }^{31}$ For SMILE, the correction of astigmatism results in an oval image of the lenticule that can be one of the key causes for asymmetry. However, the postoperative coma may also be caused by the vertical end of the Refractive Lenticular in SMILE compared with the $2.0 \mathrm{~mm}$ transition quarter in the FS-LASIS optical quarter. ${ }^{32}$ No discrepancy between SMILE and Fs-LASIK was noticed in the implemented average HOAs or SA. 
As regards the corneal asphericity; we did not discover a statistically massive distinction in the $\Delta \mathrm{Q}$ rate among both groups, however SMILE tended to produce more oblate surface, also it was reported a statistically significant more ablation of corneal surface with wave front guided ablation in comparison to flex procedure. ${ }^{30}$ There was no statistically significant difference any more as we used the LASIK aspheric Q-adjustment profile in our study.

\section{CONCLUSION}

SMILE was more effective, safe and predictable manner as Fs-LASIK. It had better outcomes in dry eye parameters at 3 months post-operative. Both techniques improved the overall corneal aberrations with no difference between both groups. SMILE probably caused additional coma aberration due to decentration, but Fs-LASIK introduced spherical aberrations, however all these adjustments had been statistically not big among all patients.

\section{REFERENCES}

1. Kamiya K, Shimizu K, Igarashi A, et al Comparison of visual acuity, higher-order aberrations and corneal asphericity after refractive lenticule extraction and wavefront guided laser-assisted in situ keratomileusis for myopia. Br J Ophthalmol 2013; 97:968-75.

2. Blum M, Täubig K, Gruhn C, et al. Five-year results of small incision lenticule extraction (ReLEx SMILE)," The British Journal of Ophthalmology 2016; 100(9):1192-5.

3. Chen X, Wang Y, Zhang J, et al. Comparison of ocular higher-order aberrations after SMILE and Wavefront-guided Femtosecond LASIK for myopia. BMC Ophthalmology 2017; 17(1):42.

4. Vestergaard A, Ivarsen A, Asp S, et al. Femtosecond (FS) laser vision correction procedure for moderate to high myopia: a prospective study of ReLEx flex and comparison with a retrospective study of FS-laser in situ keratomileusis. Acta Ophthalmol 2013; 91:35562.

5. Dos Santos AM, Torricelli AA, Marino GK, et al. Femtosecond laser-assisted LASIK flap complications. J Refract Surg 2016; 32(1):52-9.

6. Chan TC, Ng AL, Cheng GP, et al. Vector analysis of astigmatic correction after smallincision lenticule extraction and femtosecondassisted LASIK for low to moderate myopic astigmatism. Br J Ophthalmol 2016; 100(4):5539.

7. Pedersen IB, Ivarsen A and Hjortdal J. ThreeYear Results of Small Incision Lenticule Extraction for High Myopia: Refractive Outcomes and Aberrations. J Refract Surg 2015; 31:719-24.
8. Liu M, Chen Y, Wang D, et al. Clinical outcomes after SMILE and femtosecond laser-assisted LASIK for myopia and myopic astigmatism: a prospective randomized comparative study. Cornea 2016; 35(2):210-6.

9. Jiang MS, Yuan Y, Gu ZX, et al. Corneal confocal microscopy for assessment of diabetic peripheral neuropathy: a meta-analysis. $\mathrm{Br} J$ Ophthalmol. 2015; 7(3): 50-5.

10. Liu YC, Ang HP, Teo EP, et al. Wound healing profiles of hyperopic-small incision lenticule extraction (SMILE). Scientific Reports 2016; 6: 29802.

11. Kamiya K, Shimizu K, Igarashi A, et al. Visual and refractive outcomes of femtosecond lenticule extraction and small incision lenticule extraction for myopia. Am J Ophthalmol 2014; 157:128-34 e122.

12. Chan A, Ou J and Manche EE. Comparison of the femtosecond laser and mechanical keratome for laser in situ keratomileusis. Arch Ophthalmol 2008; 126:1484-90.

13. Vestergaard A, Ivarsen AR, Asp S, et al. Smallincision lenticule extraction for moderate to high myopia: Predictability, safety, and patient satisfaction. J Cataract Refract Surg 2012; 38:2003-10

14. Agca A, Demirok A, Cankaya KI, et al. Comparison of visual acuity and higher-order aberrations after femtosecond lenticule extraction and small-incision lenticule extraction. Cont Lens Anterior Eye 2014; 37:292-6.

15. Gertnere J, Solomatin I and Sekundo W. Refractive lenticule extraction (ReLEx flex) and wavefront-optimized Femto-LASIK: comparison of contrast sensitivity and high-order aberrations at 1 year. Graefes Arch Clin Exp Ophthalmol 2013; 251:1437-42.

16. Ambrósio R, Tervo T and Wilson SE. LASIKassociated dry eye and neurotrophic epitheliopathy: patho-physiology and strategies for prevention and treatment. J Refract Surg 2008; 24:396-407.

17. Yu EY, Leung A, Rao S, et al. Effect of laser in situ keratomileusis on tear stability. Ophthalmology 2000; 107:2131-5.

18. Golas L and Manche EE. Dry eye after laser in situ keratomileusis with femtosecond laser and mechanical keratome. J Cataract Refract Surg 2011; 37: 1476-80.

19. Horwath-Winter J, Vidic B, Schwantzer G, et al. Early changes in corneal sensation, ocular surface integrity, and tear-film function after laserassisted subepithelial keratectomy. $J$ Cataract Refract Surg 2004; 30: 2316-21. 
20. Toda I, Asano-Kato N, Komai-Hori Y, et al. Dry eye after laser in psitu keratomileusis. Am J Ophthalmol 2001; 132: 1-7.

21. Chao C, Golebiowski B and Stapleton F. The role of corneal innervation in LASIK-induced neuropathic dry eye. Ocul Surf 2014; 12:32-45.

22. Denoyer A, Landman E, Trinh L et al. Dry Eye Disease after Refractive Surgery. Ophthalmology 2015; 122(4): 669-76.

23. Pesudovs K. Wavefront aberration outcomes of LASIK for high myopia and high hyperopia. Journal of Refractive Surgery 2005; 21(5): S508-12.

24. Gatine D, Malet J, Hoang-Xuan T, et al. Corneal asphericity change after excimer laser hyperopic surgery: theoretical effects on corneal profiles and corresponding Zernike expansions. Investigative Ophthalmology and Visual Science 2004; 45(5): 1349-59.

25. Ye M, Liu C, Lioa R, et al. SMILE and Wavefront-Guided LASIK Out-Compete Other Refractive Surgeries in Ameliorating the Induction of High-Order Aberrations in Anterior Corneal Surface. J Ophthalmol 2016; 3(2): 43-7.

26. Oshika $\mathrm{T}$, Tokunaga $\mathrm{T}$, Samejima $\mathrm{T}$, et al Influence of pupil diameter on the relation between ocular higher-order aberration and contrast sensitivity after laser in situ keratomileusis. Invest Ophthalmol Vis Sci 2006; 47:1334-8
27. Buzzonetti L, Petrocelli G, Valente P, et al. Comparison of corneal aberration changes after laser in situ keratomileusis performed with mechanical microkeratome and IntraLase femtosecond laser: 1-year follow-up. Cornea $2008 ; 27: 174-9$.

28. Reinstein DZ, Archer TJ and Randleman JB. Mathematical model to compare the relative tensile strength of the cornea after PRK, LASIK, and small incision lenticule extraction. $J$ Refract Surg 2013; 29:454-60.

29. Vega-Estrada A, Alió JL, Arba Mosquera S, et al. Corneal higher order aberrations after LASIK for high myopia with a fast repetition rate excimer laser, optimized ablation profile, and femtosecond laser-assisted flap. J Refract Surg 2012; 28(4):689- 96.

30. Kamiya K, Shimizu K, Igarashi A, et al. Comparison of visual acuity, higher-order aberrations and corneal asphericity after refractive lenticule extraction and wavefrontguided laser-assisted in situ keratomileusis for myopia. Br J Ophthalmol. 2013; 97:968-75

31. Lombardo $\mathrm{Ma}$ and Lombardo G. Wave aberration of human eyes and new descriptors of image optical quality and visual performance. Journal of Cataract and Refractive Surgery 2010; 36(2):313-31.

32. Sekundo W, Gertnere J, Bertelmann T, et al. One-year refractive results, contrast sensitivity, high-order aberrations and complications after myopic small incision lenticule extraction (ReLEx SMILE). Graefes Arch Clin Exp Ophthalmol 2014; 252:837-43. 